\title{
How to Measure Deviation from the Kerr Initial Data: Recent Progress
}

\author{
Thomas Bäckdahl and Juan A. Valiente Kroon
}

\begin{abstract}
In these proceedings we will present recent progress concerning a construction of a geometric invariant for initial data sets for the Einstein vacuum field equations. This geometric invariant vanishes if and only if the initial data set corresponds to data for the Kerr spacetime, and thus, it characterizes this type of data. The construction was initially based on Killing spinors, but here we translate the results to tensor language. We can now handle both compact domains and domains reaching the asymptotically flat ends.
\end{abstract}

\section{Introduction}

Given a solution $\left(\mathscr{S}, h_{a b}, K_{a b}\right)$ to the Einstein vacuum constraint equations, how do we know if it is a slice of the Kerr spacetime? If not, can we measure how much it differs? These are the questions we will consider here. We will introduce a geometric invariant on the slice, which will measure this deviation from Kerr data. The invariant is constructed as an $L^{2}$-norm constructed from global information on the slice, but it only depends on information from one slice, and is therefore local in time.

It is expected that a dynamical vacuum black hole will always settle down to a Kerr black hole. To make a proper mathematical formulation of this statement, one will need a good way to measure how close data on a slice is to Kerr data. This gives a clear motivation for our work. Our invariant is coordinate independent and straightforward to compute. It is therefore well suited for studying how the non-

T. Bäckdahl ( $\varangle)$

Max Planck Institut für Gravitationsphysik, Albert Einstein Institut, Am Mühlenberg 1, 14476 Golm, Germany

e-mail: thomas.backdahl@aei.mpg.de

J. A. Valiente Kroon

School of Mathematical Sciences, Queen Mary University of London, Mile End Road, London E1 4NS, UK 
Kerrness evolves for numerically computed spacetimes. It can also be used as a tool for more theoretical work, for instance when one studies non-linear stability of the Kerr solution. For this purpose a coordinate independent integral over a slice as we have is well suited.

We have developed the theory in four papers [1-4]. Here we only make a summary and concentrate on some aspects of the problem. In the first paper [1] we present the general ideas and the invariant for general non-boosted slices with two asymptotic ends. The second paper [2] contains all technical details and generalizations so even boosted slices can be handled. The third paper [3] deals with the same problem, but only using data exterior to a surface. In the fourth paper [4] we also introduce an outer boundary so our domain becomes compact. This requires some extra conditions that we in the previous papers got from the asymptotic behaviour.

\section{Characterization of the Kerr Spacetime}

To construct the non-Kerrness invariant of initial data on a slice, we begin with a spacetime characterization of the Kerr solution. We then make a $3+1$ splitting of the equations involved. This is done in such a way that one can reconstruct the spacetime objects that we used for the spacetime characterization. Therefore, we get an initial data characterization of Kerr initial data. The initial data equations one obtains are then used to construct the non-Kerrness invariant that measures the deviation from Kerr data.

In our papers we used a spacetime characterization based on Killing spinors, but for ease of presentation we will here translate this to Killing-Yano tensors.

\subsection{Killing-Yano Tensors}

Throughout, we will assume that $\left(\mathscr{M}, g_{\mu \nu}\right)$ is an orientable and time orientable globally hyperbolic vacuum spacetime, and we let $\nabla_{\mu}$ denote the Levi-Civita connection of $g_{\mu \nu}$. Here we will use the $(-+++)$ sign convention to obtain a positive definite spatial metric. Observe that this differs from the one used in the references [1-4].

Definition 1 A conformal Killing-Yano tensor is an antisymmetric tensor $Y_{\mu \nu}=$ $Y_{[\mu \nu]}$ that satisfies

$$
\nabla_{(\mu} Y_{\nu) \lambda}=\frac{1}{3} g_{\lambda(\mu} \nabla^{\sigma} Y_{\nu) \sigma}-\frac{1}{3} g_{\mu \nu} \nabla^{\sigma} Y_{\lambda \sigma}
$$

It is called a Killing-Yano tensor if it satisfies

$$
\nabla_{(\mu} Y_{\nu) \lambda}=0
$$


Given a (conformal) Killing-Yano tensor, one automatically gets a Killing vector $\xi_{\mu}=\varepsilon_{\mu}{ }^{\nu \gamma \lambda} \nabla_{\nu} Y_{\gamma \lambda}$ of the spacetime. We also get an integrability condition that will strongly restrict the Weyl tensor. In the tensorial picture, this condition is given by

$$
0=-C_{[\mu \nu]}^{\delta}{ }_{[\lambda} Y_{\rho] \delta}-C_{[\lambda \rho]}^{\delta}\left[\mu Y_{\nu] \delta}+C_{[\mu \nu][\lambda}^{\delta} Y_{\rho] \delta}+C_{[\lambda \rho][\mu}^{\delta} Y_{\nu] \delta} .\right.
$$

The spinorial version of the above condition is simpler. From it, it is easy to conclude that the spacetime has to be of Petrov type $D, N$ or $O$.

\subsection{Spacetime Characterization}

We have the following theorem which is a translation of Theorem B.3 in [3]

Theorem 1 A smooth spacetime $\left(\mathscr{M}, g_{\mu \nu}\right)$ is locally isometric to the Kerr spacetime if and only if the following conditions are satisfied:

(i) there exists a Killing-Yano tensor $Y_{\mu \nu}$, with associated Killing vector $\xi_{\mu}$;

(ii) the spacetime $\left(\mathscr{M}, g_{\mu \nu}\right)$ has a stationary asymptotically flat 4-end with nonvanishing mass in which $\xi_{\mu}$ tends to a time translation.

\subsection{Initial Data Characterization}

To get an initial data characterization, we make a $3+1$ splitting of the conformal Killing-Yano equation and the integrability condition to obtain the (4a)-(4b) below. After some work we see that one can propagate solutions to this system to obtain a conformal Killing-Yano tensor of the spacetime. See [2] and [4] for details.

Theorem 2 Let $\left(\mathscr{S}, h_{a b}, K_{a b}\right)$ be a vacuum initial data set, where $\mathscr{S}$ is a Cauchy hyper-surface. The development of the initial data set will have a conformal KillingYano tensor in the domain of dependence of $\mathscr{S}$ if and only if

$$
\begin{aligned}
\zeta_{a b} & \equiv D_{(a} \kappa_{b)}-\frac{1}{3} h_{a b} D_{d} \kappa^{d}-i \varepsilon_{(a}{ }^{d l} K_{b) d} \kappa_{l}=0, \\
F_{a b} & \equiv-C_{(a}{ }^{c} \varepsilon_{b) c}{ }^{d} \kappa_{d}=0,
\end{aligned}
$$

are satisfied on $\mathscr{S}$. Here, $C_{a b} \equiv E_{a b}+i B_{a b}$, where $E_{a b}$ and $B_{a b}$ are the electric and magnetic parts of the Weyl tensor. Furthermore, these conditions give a complex spacetime Killing vector. Reality of this Killing vector gives a Killing-Yano tensor.

Remark The 1-form $\kappa_{a}$ is the pull-back of $-\frac{i}{2} t^{\nu} Y_{\mu \nu}+\frac{1}{4} \varepsilon_{\mu \nu \lambda \delta} t^{\nu} Y^{\lambda \delta}$, where $t^{\mu}$ is the normal to $\mathscr{S}$ with normalization $t^{\mu} t_{\mu}=-1$. Observe that we use a different normalization in references [1-4]. The lapse and shift of the Killing vector initial data is constructed from $\kappa_{a}$ via 


$$
\zeta=D^{a} \kappa_{a}, \quad \zeta_{a}=-\frac{3 i}{2} \varepsilon_{a}{ }^{b c} D_{c} \kappa_{b}+\frac{3}{2} K_{a b} \kappa^{b}-\frac{3}{2} K^{b}{ }_{b} \kappa_{a} .
$$

The vacuum constraint equations are

$$
R=K_{a b} K^{a b}-\left(K_{a}^{a}\right)^{2}, \quad D^{a} K_{a b}=D_{b} K_{a}^{a} .
$$

The electric and magnetic parts of the Weyl tensor can be written entirely in terms of initial data via

$$
E_{a b}=-K_{a}^{c} K_{b c}+K_{a b} K_{c}^{c}-R_{a b}, \quad B_{a b}=-\varepsilon_{(a}^{c d} D_{|c|} K_{b) d} .
$$

\section{Non-Kerrness Invariant}

The idea behind our non-Kerrness invariant is to measure the $L^{2}$-norms of the left hand sides of (4a) and (4b) for a clever choice of $\kappa_{a}$. We have to choose $\kappa_{a}$ in a unique coordinate independent way, and we need it to coincide with the solution to the system (4a) and (4b) if such a solution exists. It turns out that minimizing the $L^{2}$-norm of left hand side of (4a) while specifying the asymptotic behaviour, gives rise to a good choice.

\subsection{Approximate Killing-Yano Tensors}

Let $J$ denote the $L^{2}$-norm of the left hand side of (4a), that is

$$
J=\int_{\mathscr{S}} \zeta_{a b} \bar{\zeta}^{a b} \mathrm{~d} \mu
$$

To minimize this we need to solve the corresponding Euler-Lagrange equation, which reads

$$
L\left(\kappa_{a}\right) \equiv D_{b} \zeta_{a}{ }^{b}+i \varepsilon_{a c f} K^{b c} \zeta_{b}{ }^{f}=0 .
$$

The operator $L$ is a linear second order self adjoint elliptic operator. A solution, $\kappa_{a}$, to the elliptic equation (9) is called an approximate spatial Killing-Yano tensor. Clearly, any solution to $\zeta_{a b}=0$ is also a solution to (9).

Definition 2 An initial data set $\left(\mathscr{S}, h_{a b}, K_{a b}\right)$ will be called asymptotically Schwarzschildean at an end if there is a Schwarzschild initial data set with the same asymptotic expansion up to and including the mass order term. 
For non-boosted data and asymptotically Cartesian coordinates this condition reads

$$
\begin{aligned}
h_{i j} & =\left(1+2 m r^{-1}\right) \delta_{i j}+o_{\infty}\left(r^{-3 / 2}\right), \\
K_{i j} & =o_{\infty}\left(r^{-5 / 2}\right)
\end{aligned}
$$

where $o_{\infty}\left(r^{\delta}\right)$ denotes functions in a weighted Sobolev space, and $m$ denotes the ADM-mass of the asymptotic end. See [2] for definitions of the Sobolev spaces and asymptotics for boosted data.

Theorem 3 Given an initial data set $\left(\mathscr{S}, h_{a b}, K_{a b}\right)$ with two asymptotically Schwarzschildean ends, there exists a smooth unique solution to (9) with the same asymptotic behaviour as the solution for Kerr from Theorem 1.

This result was proven in [2]. In [3] we replaced one asymptotic end with an inner boundary. Appropriate data for the boundary was then constructed from the Weyl tensor. Finally in [4] we replaced all asymptotic ends with boundaries so the result extends to compact domains. In the latter case however, we needed to add extra conditions on one point to replace the asymptotic conditions in Theorem 1.

Now we can define the geometric invariant. Let $\kappa_{a}$ be a solution to (9) as given by Theorem 3. With

$$
\begin{aligned}
J & =\int_{\mathscr{S}} \zeta_{a b} \bar{\zeta}^{a b} \mathrm{~d} \mu, \\
I_{1} & \equiv \int_{\mathscr{S}} F_{a b} \bar{F}^{a b} \mathrm{~d} \mu,
\end{aligned}
$$

the geometric invariant is defined by

$$
I \equiv J+I_{1}
$$

By construction $I$ is coordinate independent and non-negative. It can furthermore be verified that it is finite. More importantly we have

Theorem 4 Let $\left(\mathscr{S}, h_{a b}, K_{a b}\right)$ be a vacuum initial data set with two asymptotically Schwarzschildean ends. Let I be the invariant defined above, where $\kappa_{a}$ is the only solution to (9) with the same asymptotic behaviour as the solution for Kerr from Theorem 1. The invariant I vanishes if and only if the development of $\left(\mathscr{S}, h_{a b}, K_{a b}\right)$ is locally isometric to the Kerr spacetime.

For the proof, see Theorem 28 in [2] and Theorem B.3 in [3] together with Theorem 4 in [4]. In [3] this was extended to the case with one asymptotic end and an inner boundary, and in [4] this was worked through for compact domains. 
Acknowledgments T.B. is funded by the Max-Planck-Institute for Gravitational Physics, Albert Einstein Institut.

\section{References}

1. Bäckdahl, T., Valiente Kroon, J.: Geometric invariant measuring the deviation from Kerr data. Phys. Rev. Lett. 104, 231102 (2010). doi:10.1103/PhysRevLett.104.231102

2. Bäckdahl, T., Valiente Kroon, J.: On the construction of a geometric invariant measuring the deviation from Kerr data. Ann. Henri Poincaré 11, 1225 (2010). doi:10.1007/s00023-0100063-2

3. Bäckdahl, T., Valiente Kroon, J.: The 'non-Kerrness' of domains of outer communication of black holes and exteriors of stars. Proc. R. Soc. Lond. Ser. A 467, 1701 (2011). doi:10.1098/ rspa.2010.0535

4. Bäckdahl, T., Valiente Kroon, J.A.: Constructing 'non-Kerrness' on compact domains. J. Math. Phys. 53, 042503 (2012). doi:10.1063/1.3702569 